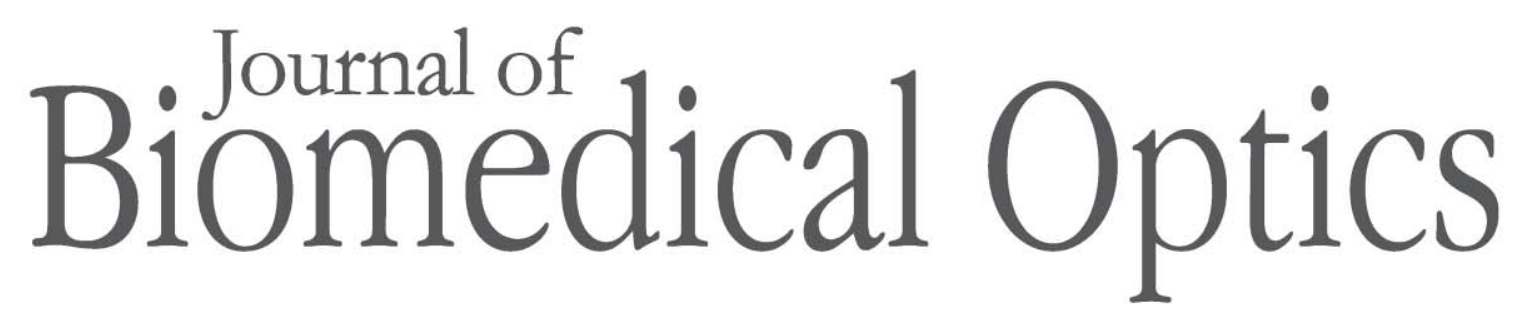

SPIEDigitalLibrary.org/jbo

\title{
Optical coherence elastography: current status and future applications
}

Cuiru Sun

Beau Standish

Victor X. D. Yang 


\title{
Optical coherence elastography: current status and future applications
}

\author{
Cuiru Sun, ${ }^{a}$ Beau Standish, ${ }^{a}$ and Victor X. D. Yang ${ }^{a, b}$ \\ ${ }^{a}$ Ryerson University, Department of Electrical and Computer Engineering, Biophotonics and Bioengineering \\ Laboratory, Toronto, Ontario M5B 2K3, Canada \\ bUniversity of Toronto, Division of Neurosurgery, Toronto, Ontario M5G 1L2, Canada
}

\begin{abstract}
Optical coherence tomography (OCT) has several advantages over other imaging modalities, such as angiography and ultrasound, due to its inherently high in vivo resolution, which allows for the identification of morphological tissue structures. Optical coherence elastography (OCE) benefits from the superior spatial resolution of OCT and has promising applications, including cancer diagnosis and the detailed characterization of arterial wall biomechanics, both of which are based on the elastic properties of the tissue under investigation. We present OCE principles based on techniques associated with static and dynamic tissue excitation, and their corresponding elastogram image-reconstruction algorithms are reviewed. OCE techniques, including the development of intravascular- or catheter-based OCE, are in their early stages of development but show great promise for surgical oncology or intravascular cardiology applications. (O 2011 Society of Photo-Optical Instrumentation Engineers (SPIE). [DOI: 10.1117/1.3560294]
\end{abstract}

Keywords: optical coherence elastography; optical coherence tomography; intravascular optical coherence elastography.

Paper 10414VR received Jul. 21, 2010; revised manuscript received Jan. 10, 2011; accepted for publication Feb. 7, 2011; published online Apr. 1, 2011.

\section{Introduction}

\subsection{Elastography}

Elastography is an experimental method and a noninvasive medical imaging technique that conveys new information about internal tissue structure and behavior under load and ultimately generates images called elastograms. ${ }^{1}$ Examples include elastograms of axial strains, lateral strains, and elastic moduli. Different degrees of stiffness are demonstrated as different shades of light and dark in an elastogram. ${ }^{2}$ Ideally, this elastography technique can extract the elastic modulus, which is a property of the constituent material describing the stiffness of an isotropic elastic material. ${ }^{3}$ There are several types of elastic moduli, where the main moduli used currently in elastography are the Young's modulus (the ratio of the uniaxial stress over the uniaxial strain) and shear modulus (the ratio of shear stress to the shear strain). Given any two of the six elastic moduli, the remaining modulus can be determined. Thus two elastic moduli can fully describe the elastic properties of a homogeneous and isotropic material.

The motivation underlying elastography stems from the fact that elastic properties of tissues are directly related to the underlying structure of the tissue and are strongly affected by pathological changes. For example, edema, fibrosis, and calcification alter the elastic modulus of the extracellular tissue matrix ${ }^{4}$ different tissues within atherosclerotic plaque have distinctive elastic properties, ${ }^{5}$ and cancerous tumors are often stiffer than benign and normal tissue. ${ }^{6}$ Elastography images contain information of local variations of the stiffness inside a region of interest and may provide additional clinical information to aid the identification of suspicious lesions and diagnosis of various

Address all correspondence to: Victor X. D. Yang, Ryerson University, Department of Electrical and Computer Engineering, Biophotonics and Bioengineering Laboratory, Toronto, Ontario M5B 2K3, Canada. Tel: 416-946-4501; E-mail: yangv@ee.ryerson.ca disease states. ${ }^{7-9}$ Currently, there are several potential applications of elastography, which include assessing breast or brain tissue for malignancy ${ }^{10,11}$ and atherosclerotic arteries for vulnerability to myocardial infarction. ${ }^{12}$

\subsection{Optical Coherence Elastography}

Optical coherence tomography (OCT)-based elastography, which is termed optical coherence elastography (OCE), ${ }^{13}$ was first introduced by $\mathrm{Schmitt}^{14}$ in 1998. OCE shows great potential for micron and submicron imaging applications because it benefits from the high resolution of OCT, ${ }^{15}$ while additionally providing the elastic properties of the sample. OCT provides structural images approaching histology, where the microstructures of biological tissues can be quantified based on the optical backscattering properties within the imaging region of interest. This beneficial high-resolution, noninvasive imaging modality allows OCE to evaluate the mechanics of intact tissue on a scale that cannot be offered by elastographies produced by the competing imaging modalities of ultrasound ${ }^{3,16-18}$ or MRI (magnetic resonance imaging). ${ }^{19,20}$ Recent advances of these technologies are pushing the resolution to higher values where high-frequency ultrasound ${ }^{21}$ can reach $30-70 \mu \mathrm{m}$ and magnetic resonance microscopy ${ }^{22}$ has improved imaging resolution to $\sim 120 \mathrm{um}$. However, OCT has inherently high resolution, 1-10 $\mu \mathrm{m}$ in vivo; therefore, OCE has the potential to link microstructural data with biomechanical and functional information. In the last decade, several studies have demonstrated the ability of OCE to provide valuable information about the micromechanical properties of tissue. OCE has been used to investigate the microscopic deformation of gelatin tissue phantoms, porcine muscle, and human skin as a function of depth

1083-3668/2011/16(4)/043001/12/\$25.00 (C) 2011 SPIE 
under compressive loads; ${ }^{23}$ define the properties of collagen organization in musculoskeletal tissue ${ }^{24}$ provide the material properties of the cornea; ${ }^{25}$ develop strain maps of engineered tissue and a Xenopus tadpole at different stages of embryonic development; ${ }^{13}$ and define tumor and normal adipose human breast tissue borders. ${ }^{26}$ Finally, one of the current research directions in OCE imaging has been to develop intravascular OCE for rupture-prone (vulnerable) plaque detection. ${ }^{27}$

\subsection{Intravascular Elastography for Atherosclerotic Plaque Detection}

The main components of atherosclerotic plaque include a large extracellular necrotic core with a thin fibrous cap infiltrated by macrophages, all of which have varying biomechanical properties. ${ }^{28,29}$ Rupture of the cap induces the formation of a thrombus, which may obstruct the coronary artery, causing an acute heart attack and frequently results in patient death. ${ }^{30,31}$ Detailed characterization of the arterial wall biomechanics may provide complementary information to quantify lesion stability. Both in vitro and in vivo studies have revealed that strain is higher in fatty tissue components than in fibrous plaques. ${ }^{32}$ The presence of a high-strain area that is surrounded by a low-strain region has a high predictive power to identify the vulnerable plaque with high sensitivity and specificity. ${ }^{33}$ Thus, changes in the mechanical properties of the tissue can be detected, quantified, and correlated with clinical symptoms and inflammation markers.

Intravascular elastography is particularly used to correlate elastograms with histological characteristics of the blood vessel wall. The ultrasound community has made significant progress in developing intravascular ultrasound (IVUS) elastography, ${ }^{34}$ which has been successfully used to extract arterial radial strains with a spatial resolution of $200 \mu \mathrm{m}$. Schmitt et al. ${ }^{35}$ have used ultrasound elastography within carotid walls induced by the natural cardiac pulsation, and coupled to a simple inverse problem, to recover the wall elastic modulus at the blood pulsatility frequency $(1 \mathrm{~Hz})$. This was the first in vivo axial strain and shear distributions of atherosclerotic carotid arteries measurement. In 2008, Schmitt et al. ${ }^{36}$ made further progress by adapting the dynamic microelastography method and formulating an inverse problem to study the radial viscoelasticity of the vessel wall. Although this has provided some success in plaque identification, there exist several limitations because vulnerable atherosclerotic plaques have structural components (e.g., fibrous caps) on the order of 50-200 $\mu \mathrm{m}$, which lie below the resolvable limit of the IVUS imaging system. OCT is a potential technical solution to overcome these hurdles because it is not limited, in this regard, due to its inherent high resolution and provides noninvasive near-cellular-level imaging for plaque quantification.

An important application of intravascular $\mathrm{OCT}^{37,38}$ is the detection of thin-cap fibroatheroma (TCFA), the most important type of vulnerable plaques. Xu et al. ${ }^{39}$ have addressed fundamental issues that underlie the tissue characterization of OCT images obtained from coronary arteries. With high spatial resolution and, thus, the ability to visualize structures on the size scale of thin fibrous caps, intravascular OCE has the potential to provide high-resolution characterization of strains in tissue lying within $1.0-1.5 \mathrm{~mm}$ of the lumen interface, the region most susceptible to plaque disruption. ${ }^{27}$ Chau et al. ${ }^{32}$ have used OCT as a basis for finite element analysis and obtained results comparable to histology methods for stress and strain analysis of atherosclerosis. Their results suggest that OCT-based finite element analysis may be a powerful tool for investigating coronary atherosclerosis and detecting vulnerable plaque. The capability of OCE to resolve TCFA is advantageous when compared to competing technologies, such as IVUS. Chan et al. ${ }^{27}$ and Khalil et al. ${ }^{40,41}$ have performed several studies highlighting the benefits of intravascular OCE, which may play an important role in the elasticity estimation of vulnerable plaques and provide patient risk stratification for appropriate treatment or vascular targeted therapies.

\subsection{Technique Development of Optical Coherence Elastography}

When considering OCE as a diagnostic tool, two important issues must be considered. The first is the mechanical design of a loading system because an external load is usually required to excite tissues for a certain deformation. Therefore, a loading system must be able to apply a known and reproducible stress to avoid artifacts from nonuniform stress distributions, be easy to use, and be compatible with the existing time-domain (TD) or frequency-domain (FD) OCT systems. The second issue relates to data analysis for reconstruction of tissue elastograms, which is a process of combining imaging, elastography, and computational modeling to build maps of soft-tissue mechanical properties. In general, soft tissues are viscoelastic, anisotropic, and incompressible. No biological soft tissue exhibits a truly elastic response, but under some conditions, the assumption of linear elasticity is both reasonable and useful. ${ }^{42}$ For example, when the deformation is small $(<0.1 \%)$, the nonlinearity of the elastic constants of soft tissues can be neglected.$^{25}$ Under the assumption that the tissue is linear elastic, isotropic, and subjected to a constant stress field, the strain field could be interpreted as a relative measure of elasticity distribution, where the strain is large in a compliant (soft) tissue, and small in a rigid (hard) tissue. ${ }^{43}$ However, for anisotropic materials, such as arteries with asymmetric geometries and the presence of plaques leading to a nonhomogeneous arterial wall stress field, the material property identification is much more complicated than for isotropic materials.

Inverse problem-solving ${ }^{43,44}$ methods are usually developed to solve for unknown material properties from measured displacements in anisotropic materials. Prior to solving the inverse problem, constitutive relations need to be defined to describe the behavior of individual tissues depending on the conditions of interest, including the parameters of the loading profile, material properties, geometric feature of the structure, boundary conditions, and/or a combination of these behaviors. Then, if a set of experimentally measured data (displacement, velocity, strain, natural frequency, etc., obtained by OCE) are available for the structure of interest, the material property can be characterized by minimizing the sum of the squares of the errors or discrepancy between the experimental and computed structure behavior data. ${ }^{45,46}$ Elastograms of strain or modulus can then be reconstructed. Although hurdles to widespread clinical acceptance exist, short-term engineering solutions are being investigated, such that OCE may play a very important role as a diagnostic tool in the near future. 
This review summarizes recent advances in OCE, including technical issues involved in the design of loading schemes associated with OCT platforms along with theoretical analysis for displacement, strain, and elastic moduli elastogram reconstruction. Clinical applications including intravascular OCE are presented, followed by a discussion of the future role of OCE in terms of technical development and clinical utility.

\section{Optical Coherence Elastography Approaches}

Karimi et al. ${ }^{47}$ demonstrated an overall flowchart of the elastography process shown as Fig. 1, where the main steps include the following:

(1) Identify the structural characteristics of tissue (i.e., isotropic or anisotropic) and establish an appropriate theoretical framework with suitable restrictions on the possible constitutive relations connecting applied stresses or forces to strains or deformations.

(2) Design appropriate experimental methods, including the detection of the natural deformation of tissue due to factors such as blood flow or external excitation based on the theoretical framework. Displacement or strain elastograms can then be obtained through medical imaging modalities, including MRI, ultrasound, and OCT.

(3) Obtain a reliable stress-strain constitutive relation and elastic modulus elastograms. This process is based on an iterative scheme to minimize the difference between the strain obtained by the constitutive model and experimental results.

The displacement or strain elastograms are usually obtained through directly processing structural images obtained by biomedical imaging modalities, such as ultrasound or OCT, while elastic moduli elastograms are reconstructed by further calculations. Similar to untrasound elastography, external forces are usually applied to deform soft tissues although ultrasound, specifically high-frequency ultrasound, can use its interrogating acoustic radiation force to stimulate soft tissues. There are two particular types of stimuli, static/quasi-static and dynamic stimuli. An OCE system includes two main subsystems: an OCT system and a loading system used to deform biological tissues. The following sections review OCE systems and the corresponding algorithms for elastogram reconstruction.

\subsection{Static or Quasi-Static OCE Method}

OCE takes advantage of the short coherence lengths of broadband light sources to image micron-level displacement and strain distributions of individual tissue elements as they are stretched, compressed, or vibrated by an external force. ${ }^{48}$ When static or quasi-static compression is applied to tissue, the majority of OCE techniques reported to date rely on speckle tracking ${ }^{49}$ to estimate the relative motion of subsurface structures imaged under different applied forces. The following sections present and discuss these OCE techniques in detail.

\subsubsection{Traditional Cross-Correlation-Based Method}

The cross-correlation-based OCE methodology can work for both the TD and FD OCT platforms. Typically, a sequence of B-scan OCT images are acquired before and after deformation of an object in conjunction with a loading system. The loading system uses a piezoelectric transducer attached to a glass plate, through which the OCT imaging beam is focused. A computer-controlled translation stage moves a load cell and applies necessary compression to the sample against the fixed glass slide, ${ }^{13,50}$ so that the force applied to the tissue in the axial direction ( $z$ direction) can be known from the load cell and the actual displacement of the sample can be acquired from the translation stage. Displacements are then calculated by correlating image sets obtained prior to and immediately following the

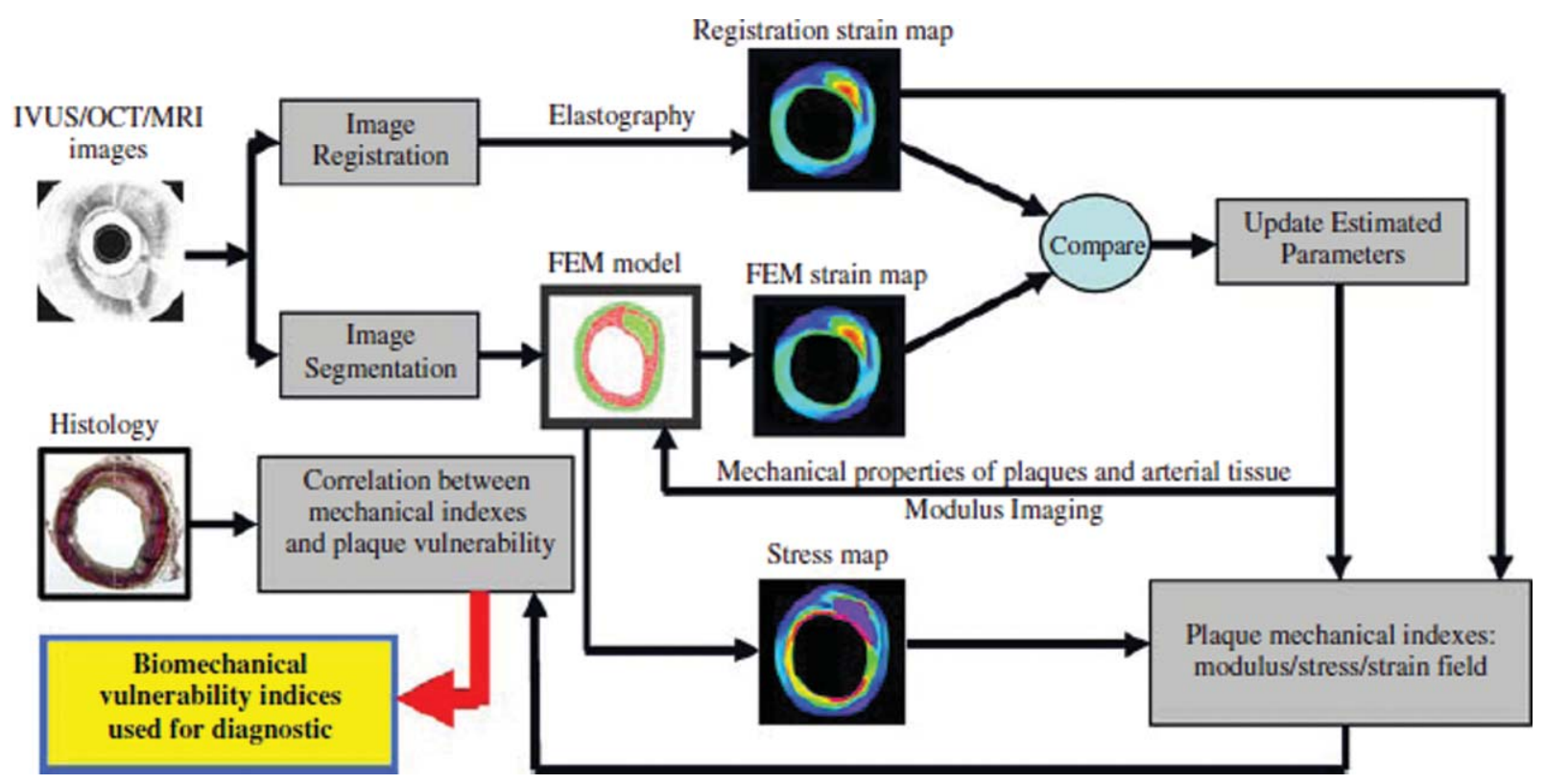

Fig. 1 Overall flowchart of the elastography and parameter estimation procedures for arterial plaque characterization (Ref. 47). Reproduced with permission from Springer-Verlag, Heidelberg. 
application of the load using Eq. (1) or equivalent forms of 2-D cross-correlation equations,

$$
\rho\left(x^{\prime}, z^{\prime}\right)=\frac{\sum_{x, z}\left[I_{1}(x, z)-\overline{I_{1}}\right]\left[I_{2}\left(x-x^{\prime}, z-z^{\prime}\right)-\overline{I_{2}}\right]}{\sqrt{\sum_{x, z}\left[I_{1}(x, z)-\overline{I_{1}}\right]^{2}\left[I_{2}\left(x-x^{\prime}, z-z\right)-\overline{I_{2}}\right]^{2}}},
$$

where $\rho\left(x^{\prime}, z^{\prime}\right)$ is the correlation coefficient, which is a function of displacements $x^{\prime}$ and $z^{\prime}$ within a predefined subset; $I_{1}(x, z)$ is the pixel intensity of a point $(x, z)$ and $I_{2}\left(x-x^{\prime}, z-z\right)$ is the pixel intensity of the point on the deformed image; $x$ is the transverse location, and $z$ is the axial (depth) location within the images. $\overline{I_{1}}$ and $\overline{I_{2}}$ are the average subset pixel values. From the resultant deformation pattern, strain images are obtained by numerically differentiating the axial displacement component assuming the applied stress was uniform or had a known distribution. In this case, the 2-D cross-correlation is applied to the $x z$ plane (B-scan of OCT) instead of the $y z$ or $x y$ plane because B-scans are recorded in real time, whereas images in other directions can only be obtained when the 3-D OCT scan is finished. Because of the well-developed cross-correlation algorithms, researchers have shown interest in cross-correlation-based OCE methods (Schmitt, ${ }^{14}$ Duncan and Kirkpatrick, ${ }^{49}$ Ko et al., ${ }^{51}$ Rogowska et al. $^{52}$ ).

Cross-correlation-based OCE has shown promise as a technique to provide additional contrast for disease detection. However, several hurdles exist that may limit its usefulness and adoption into daily imaging procedures for diagnosis, disease screening, or progression monitoring. First, correlation-based algorithms are based on the maximization of a correlation coefficient that is determined by examining pixel-intensity array subsets on two or more corresponding images. During the correlation calculation, the size of the correlation window must be chosen carefully. If the window is too small, then the displacement results may not be accurate. However, large windows tend to average out the difference in displacements of small features in images and decrease the ability of speckle tracking to make microstructural assessments. This is a time-consuming highcomplexity computational procedure, making real-time measurements and characterization difficult. Second, it is required that the resultant deformation of tissue must be large enough, where the peak of correlation coefficient shift can be quantified, but small enough such that the correlation of the speckle patterns does not lie within the potential sources of measurement error: (i) excessive surface displacement (either in-plane, out-ofplane, or rotational) due to vibration, (ii) excessive surface tilt, or (iii) plastic deformation, which may induce speckle-pattern decorrelation. ${ }^{53}$ Thus far, strain is calculated from the gradient of assessed displacement fields and the differentiation operation has a high sensitivity to local errors. Thus, cross-correlation approaches are not applicable when the deformation is either very large or very small.

Kirkpatrick et al. ${ }^{50}$ used a maximum-likelihood estimation method to deal with small deformations, by which the local velocity of every pixel point in the image was assessed and the strain elastograms were then obtained. Instead of using crosscorrelations, they also proposed a phase-sensitive OCE to process large deformations.

\subsubsection{Phase-Sensitive Optical Coherence Elastography}

Phase-sensitive OCE (PSOCE) is based on the measurement of phase changes induced by tissue motions in complex OCT images. Kirkpatrick et al. ${ }^{50}$ and Wang et al. ${ }^{54}$ developed a tissue Doppler OCE (tDOCE) approach to obtain large movement of speckles from Doppler frequency shift induced by instantaneous tissue motion. Theoretical basis of tDOCE is similar with Doppler OCT. ${ }^{55,56}$ In the spectral domain, the spectral density of combined reference and sample beams is ${ }^{50}$

$$
\begin{aligned}
I_{d}(v)= & S(v)\left[1+\int R(\tau) d \tau+2 R_{\text {self }}\right. \\
& \left.+2 \int \sqrt{R(\tau)} \cos \left(2 \pi v \tau+\phi_{0}\right) d \tau\right],
\end{aligned}
$$

where $v$ is the frequency of the light source, $R(\tau)$ is the normalized intensity backscattered from the scatter at time $\tau, \phi_{0}$ is the initial phase value at $\tau=0$, and $S(v)$ is the spectral density of the light source. The fourth term in the brackets represents the spectral interference between the sample and the reference and is the term that contributes to the OCT signal. Discard the first three terms and perform Fourier transform; the depth information regarding the local scatters in the sample is obtained:

$$
I(z)=A(z) \exp [-i \Phi(z)] .
$$

If the scatterer at position $z$ is translated by a distance $\Delta d(z)$ during the time interval $\Delta t$ between two successive A-scans, then the induced phase change will be

$$
\Delta \Phi(z, t)=2 n k \Delta d(z),
$$

where $k$ is the wavenumber of the light source and $n$ is the refractive index of the sample. The depth-resolved tissue velocity $v(z, t)$ in the beam direction could then be obtained from the phase change, $\Delta \Phi(z, t)$ by

$$
v(z, t)=\frac{\Delta d}{\Delta t}=\frac{\Delta \Phi(z, t) \lambda}{4 \pi n \Delta t},
$$

where $\lambda$ is the wavelength. The strain rate $\dot{\varepsilon}(z, t)$ can be obtained by

$$
\dot{\varepsilon}(z, t)=\frac{v(z, t)}{z_{0}}=\frac{\Delta \Phi(z, t) \lambda}{4 \pi n z_{0} \Delta t},
$$

where $z_{0}$ is the initial depth of the sample before the tissue movement. The instantaneous tissue deformation and strain rate could then be imaged in real time, and the total displacement and strain over a time period can be obtained by integration of the instantaneous displacement and strain over the elapsed time. This approach was expanded to detecting phase change between successive OCT-B scans so that small deformation can also be measured ${ }^{57}$ tDOCE is faster than cross-correlation-based OCE methods, but the sensitivity is fundamentally limited by the phase sensitivity of the OCT system. Example images of velocity, displacement, strain rate, and cumulative strain maps of a phantom are illustrated in Fig. $2,{ }^{57}$ where the phantom was compressed repeatedly by a triangular wave.

The techniques mentioned above can obtain deformation or strain elastograms, which may be enough to show the stiffness difference between healthy and diseased tissues in scenarios such as cancer diagnosis. However, for the case of atherosclerotic plaque characterization, the absolute value of modulus of 

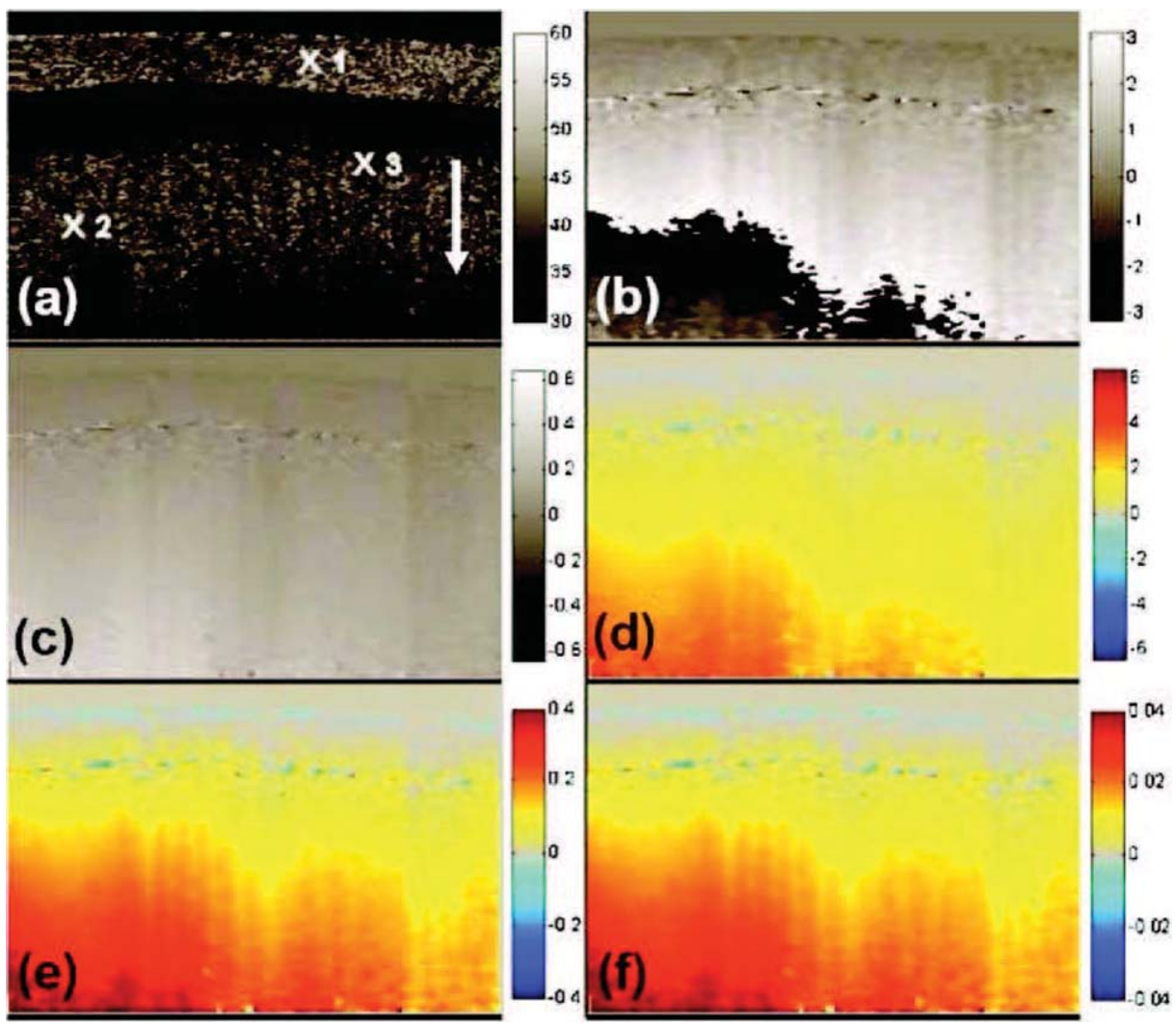

Fig. 2 Characterization of the tissue mechanical properties of a bilayer phantom at the time instant of $t=5 \mathrm{~s}$ when it was subjected to a slow dynamic compression: (a) OCT structural image (in decibels), (b) wrapped phase map determined by the PSOCE system (radians), (c) instant tissue deformation (in microns) map calculated from (b) after the application of unwrapping algorithm, (d) localized velocity map (in microns per second), (e) instant strain rate map (in percent per second), and (f) instant strain map (in percent) (Ref. 57). Reproduced with permission from The American Institute of Physics.

elasticity needs to be known. Moreover, as intrinsic mechanical properties of materials, the elastic moduli are thought to be more advantageous for wider clinical applications. ${ }^{1}$ Inverse problem approaches can be applied to aid the reconstruction of distributed elastic moduli elastograms.

\subsubsection{Tissue Elasticity Reconstruction and Inverse Problem}

Traditionally, elastography imaging protocols typically assume a "constant stress field," implying that an elastogram is equivalent to a modulus image. If the applied force is known and the strain is obtained from OCE, then the Young's modulus can be estimated, assuming a tissue is uniform, incompressible, and an isotropic linear elastic material. With the application of uniformly applied stress in the axial direction, the local stress is equal to the applied stress (constant throughout the sample), which can be calculated to yield the Young's modulus: ${ }^{52}$

$$
E=\frac{\sigma}{\varepsilon}
$$

where $E$ is the Young's modulus, $\sigma$ is stress, and $\varepsilon$ is strain. Researchers use this simple, direct linear elastic, isotropic model for two main reasons. First, elasticity estimation is a discrete numerical effort, where elasticity values are assigned to individual elements based on displacement maps generated via image processing. Second, unlike a nonlinear constitutive model, elasticity estimations provide a comprehensive map of mate- rial properties. ${ }^{40}$ However, as previously mentioned, biological tissues are anisotropic and their response to specific external excitation has a viscous as well as an elastic component. Therefore, the determination of both elastic and viscoelastic properties of soft tissues is important for full characterization of tissue. In this case, a more elaborate solution strategy is required to solve the inverse problem of elastic modulus imaging.

Karimi et al. ${ }^{47}$ have performed sophisticated attempts on using inverse problem approaches to generate elastic moduli elastograms considering tissue's nonlinear behavior. Following the flowchart shown in Fig. 1, they implemented the Mooney-Rivlin model, ${ }^{58}$ a hyperelastic material model, to estimate the mechanical behavior of the corresponding regions in their finite element method (FEM) model of an aortic specimen, including normal vessel wall, fibrous plaque, and the presence of lipid pools. An iteration scheme, multipoint scheme, and a genetic algorithm are used for material parameters estimation by minimizing the difference between the strain map obtained with elastography and the strain map calculated from the FEM model. The inverse problem technique ${ }^{59}$ has experienced initial preclinical success in several areas, where the biomechanical properties of tissue may be used to image and quantify disease formation. For example, Sridhar et al. ${ }^{60}$ applied inverse problem solutions to investigate how disease-related changes to molecular bonding within stromal tissues affect the broad spectrum of viscoelastic responses ${ }^{61}$ and proposed a new approach to minimize the meansquared error between computed and measured displacements. 
The greatest potential for the inverse problem technique aided OCE lies in patient risk stratification for management of vulnerable plaques. Floch et al. ${ }^{62}$ summarized different methods to constrain the optimization algorithm of solving inverse problems and studied the preconditioning of the algorithm based on the best estimation of the plaque component contours. However, technical investigations into the meaning of associated parameters, such as the role of the magnitude of the regularization parameter and extensive in vivo testing, are required prior to the acceptance of involving inverse problem approaches in standard clinical imaging scenarios.

\subsection{Dynamic Optical Coherence Elastography Method}

In dynamic excitation, samples are excited by various mechanical wave forms, where the distributions of dynamic displacements (time or space) within a small region of the samples are imaged to capture dynamic OCE signals. These OCE systems have high resolution in both axial and transverse directions with the major advantage of not requiring a cross-correlation algorithm.

Liang et al. ${ }^{26,63,65}$ and Xing and Boppart ${ }^{64}$ proposed a dynamic optical coherence elastography method in which tissue was excited by optomechanical waves, such as a step or sinusoidal wave form or acoustic pressure, coaxially with the optical beam, with a piezoelectric rod actuator operating in a frequency range from a $100 \mathrm{~Hz}$ to kilohertz. The Young's moduli were then extracted from the detected phase-resolved signals. In detail, the sample and mechanical wave driver were modeled as a Voigt body, via the following equation: ${ }^{63}$

$$
m \ddot{x}+\left(\gamma_{0}+\gamma\right) \dot{x}+\left(k_{0}+k\right) x=F-m g,
$$

where $x$ is the displacement of samples; $k_{0}$ and $\gamma_{0}$ are the spring constant and the damping parameter for the mechanical wave driver, respectively; $k$ and $\gamma$ are the spring constant and the coefficient of viscosity of the samples, and $m$ denotes the mass of sample stage and the samples. As described in the previous section, the phase variation of OCT signals is related to displacement of samples being measured based on Eq. (4), so the phases obtained from OCT can be fitted to the solution of Eq. (8). During the fitting process, parameters in Eq. (8) are solved. Then the elastic modulus of the sample can be calculated. Using this technique, an elasticity map of tissue containing both tumor and normal adipose breast tissue were obtained and displayed in Fig. $3 .^{26}$ The measured elasticity map is shown in Fig. 3(c), where elasticity differences on the tumor side of the OCE map are observed.

Acoustic radiation force are currently popularly used to generate an internal load noninvasively. ${ }^{66}$ Liang et al ${ }^{67}$ presented an internal mechanical excitation method using acoustic radiation forces and developed an acoustomotive OCE based on the similar mechanical model introduced above. The acoustomotive OCE has the potential for imaging biomechanical properties in vivo. However, using these dynamic methodologies, every pixel in the OCE image or biomechanical map requires waveequation fitting and, as a result, the processing time is too long for real-time applications. In addition, the feasibility of accurately detecting vibration amplitude in the presence of lateral (a)

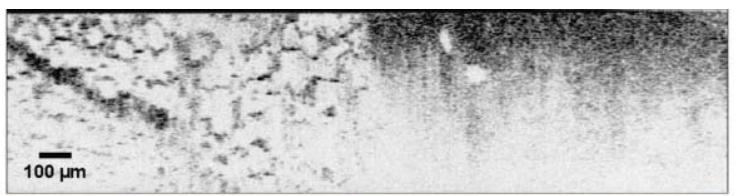

(b)

(d)

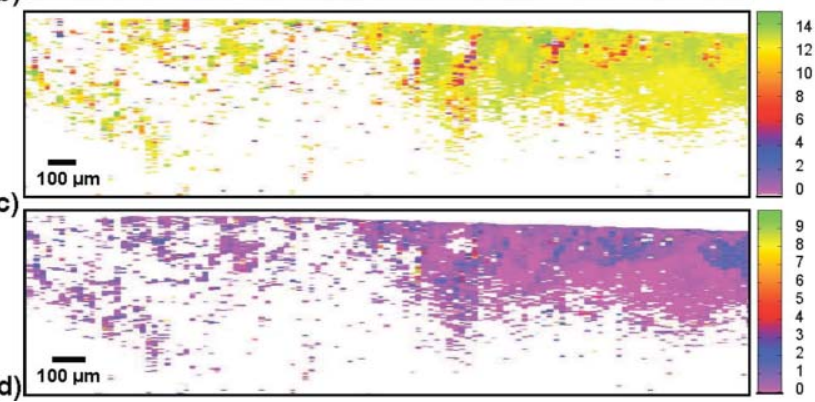

Fig. 3 Phase-resolved OCE map of human breast tissue elasticity. (a) B-mode OCT image of breast tissue. The left side of (a) represents the adipose tissue while the right side of (a) represents the tumor tissue. (b) Histology image corresponding to (a), (c) map of elasticity by sinusoidally driven phase-resolved OCE, and (d) error map of elasticity by sinusoidally driven phase-resolved OCE. Unit for color bar is $\mathrm{kPa}$ (Ref. 26). Reproduced with permission from OSA. (Color online only.)

scatterer motion, such as when shear waves are excited, needs to be investigated further for applicability in this model.

Adie et al. ${ }^{68}$ proposed an OCE technique based on analysis of the dynamic interferometry caused by sample excitation at audio frequencies. This approach is suitable for quantitative measurement of tissue elastic properties in the $100 \mathrm{~Hz}$ to kilohertz frequency range, which matches the typical frequency response range of many biological tissues. The theoretical basis of this method is to expand the detected photocurrent signal of interest as a series of Bessel functions resulting in

$$
\begin{aligned}
i_{1}(z, t)= & 2 \rho \sqrt{I_{\mathrm{R}} I_{\mathrm{S}}}\left(\operatorname { c o s } \phi _ { \mathrm { DC } } \left[J_{0}\left(\phi_{S}\right)\right.\right. \\
& \left.+2 \sum_{n=1}^{\infty} J_{2 n}\left(\phi_{S}\right) \cos (4 \pi n \Omega t)\right] \\
& \left.-\sin \phi_{\mathrm{DC}}\left\{2 \sum_{n=0}^{\infty} J_{2 n+1}\left(\phi_{\mathrm{S}}\right) \sin [2 \pi(2 n+1) \Omega t]\right\}\right),
\end{aligned}
$$

$$
\begin{aligned}
i_{2}(z, t)= & 2 \rho \sqrt{I_{\mathrm{R}} I_{\mathrm{S}}}\left(\operatorname { s i n } \phi _ { \mathrm { DC } } \left[J_{0}\left(\phi_{S}\right)\right.\right. \\
& \left.+2 \sum_{n=1}^{\infty} J_{2 n}\left(\phi_{S}\right) \cos (4 \pi n \Omega t)\right] \\
& \left.+\cos \phi_{\mathrm{DC}}\left\{2 \sum_{n=0}^{\infty} J_{2 n+1}\left(\phi_{\mathrm{S}}\right) \sin [2 \pi(2 n+1) \Omega t]\right\}\right),
\end{aligned}
$$


where $J_{n}$ is the $n$ 'th-order Bessel function, $\Omega$ is the frequency of the harmonic excitation, $I_{\mathrm{S}}$ and $I_{\mathrm{R}}$ are the sample and reference optical intensities, respectively, $\phi_{\mathrm{DC}}$ is the quasi-static interferometric phase governed by the mean axial position of the scatterer, $\rho$ is the detector responsivity, and $\phi_{\mathrm{S}}$ is expressed as

$$
\phi_{\mathrm{S}}=\frac{4 \pi}{\lambda} d(z, \Omega)
$$

where $\lambda$ is the mean optical wavelength in the medium, $d$ is the local, generally frequency-dependent, axial displacement amplitude so the mapping of $d$ represents the OCE displacement elastogram. It can be seen that $d$ can be determined from an interferometric measurement of $\phi_{\mathrm{S}}$, which can in turn be achieved through the piecewise mapping of the measured ratios of Bessel harmonic terms to the vibration amplitude based on Eqs. (9) and (10). This method however also involves extensive calculations to extract the vibration harmonics and is prone to noise. Moreover, elastograms of elastic moduli must be calculated from the obtained displacement based on the assumption of uniformly applied stress or using inverse problem-solving methods.

Because dynamic OCE is an active area of research, several additional techniques are in the beginning stages of feasibility demonstration. Huang et al. ${ }^{69}$ used a pressure-controlled air jet as an indenter to compress the soft tissue from the same side of imaging, in a noncontact manner and utilize the OCT signals to extract the deformation. Kennedy et al. ${ }^{70}$ have designed an appropriate sample arm based on a ring actuator and also enabled excitation and imaging to be achieved from the same side of the sample. Oldenburg and Boppart ${ }^{71}$ have proposed a method using embedded magnetomotive nanotransducers to excite soft tissues. However, experimental and computational techniques are still needed to further improve all of these techniques. Because of acquisition speed and the need for coaxial optical beam and vibration excitation, the in vivo application of dynamic OCE are limited to exposed skin, such as human hands.

As previously mentioned, OCE shows great advantage over ultrasound elastography on vascular disease diagnosis. However, for applications of OCE to vascular tissue characterization, special processing methodologies are usually required.

\section{Intravascular Optical Coherence Elastography}

\subsection{Variational Method}

Chan et al. ${ }^{72}$ proposed a variational method to improve deformation and strain estimation in OCE. The variational method takes a data-driven approach in which velocity filtering takes place during the correlation maximization process itself. In this manner, all the available correlation data are taken into consideration in the robust velocity estimation. Specifically, the variational method incorporates prior knowledge about velocity fields in the pulsating arterial wall, where the overall variational energy functional is

$$
E[V(x, y)]=a E_{\mathrm{D}}[V(x, y)]+b E_{\mathrm{S}}[V(x, y)]+c E_{\mathrm{I}}[V(x, y)]
$$

$V(x, y)$ is the velocity field, $E_{\mathrm{D}}[V(x, y)]$ is data fidelity, $E_{\mathrm{S}}[V(x, y)]$ is strain field smoothness, and $E_{\mathrm{I}}[V(x, y)]$ is arterial wall incompressibility. These three terms are combined based on a certain weight: $a, b$, and $c$. When the overall variational energy is minimized, the desired velocity field is obtained. Following the velocity estimation, the deformation matrix can then be calculated at every point within the OCE imaging volume.

The velocity estimation of an intact aortic segment was obtained experimentally by mounting the specimen horizontally and applying mechanical load (lateral direction) with a onedimensional translation micromanipulator to approximate circumferential stretching. This method produced improved results when compared to the traditional cross-correlation method, but a priori knowledge about arterial wall behavior was required for robust estimation of tissue motion and strain. These constraints may limit the overall usefulness of this technique in a clinical scenario based on the inhomogeneity of human tissue.

In 2005, Khalil et al. ${ }^{40}$ improved their OCE technique with the introduction of the nonlinear least-squares method, which minimizes the difference between computed and measured mechanical responses of soft tissue to estimate elasticity fields of soft tissues. This was an important step toward gaining quantitative elasticity information from in vivo OCT imaging of coronary arteries.

In 2006, the inverse elasticity problem approach ${ }^{73}$ was used comprising of FEM-derived mechanics directly within the image registration, which is the process of transforming the different sets of data into one coordinate system. Using a gradient-based search algorithm, tissue elasticity images were reconstructed given the internal tissue displacements based on subsequent imaging as the tissue is deformed. Results of in vitro OCE measurement of a porcine aorta injected with lipid are shown in Fig. 4. A fatty region was identified at the center of the arterial wall, showing a higher contrast in both the elastogram and the modulus image, further demonstrating the potential of OCE in vascular disease diagnosis by characterizing atherosclerotic plaque.

\subsection{Optical Coherence Tomography Alternating-Line Elastography}

To measure the radial displacement of vascular tissue, van Soest et al. ${ }^{74,75}$ developed a catheter-based OCT alternatingline elastography (OCTALE) technique. It employs the correlation between the overlapping point-spread functions of adjacent A-scans, instead of subsequent B-mode imaging. The B-mode data are then separated into the odd and even A-scans, where acoustic forces are applied synchronously with the A-scan rate of the OCT system. This alternated sequence of A-scans, correlating to a low- or high-pressure scenario, yields odd and even displacement fields. The OCE image can be calculated by optimizing a one-dimensional cross-correlation function ${ }^{75}$

$$
\rho_{i}(\delta)=\frac{\sum\left[I_{b}\left(r_{i}\right)-\left\langle I_{b}\left(r_{i}\right)\right\rangle\right] \cdot\left[I_{\mathrm{s}}\left(r_{i}+\delta\right)-\left\langle I_{\mathrm{s}}\left(r_{i}+\delta\right)\right\rangle\right]}{\sqrt{\sum\left[I_{b}\left(r_{i}\right)-\left\langle I_{b}\left(r_{i}\right)\right\rangle\right]^{2} \cdot \sum\left[I_{\mathrm{s}}\left(r_{i}+\delta\right)-\left\langle I_{\mathrm{s}}\left(r_{i}+\delta\right)\right\rangle\right]^{2}}},
$$

where $\delta$ is the radial lag. Angular brackets denote averaging over a window of $m$-lines by $n$ pixels. The tissue displacement is obtained when the cross-correlation coefficient $\rho$ attains its 


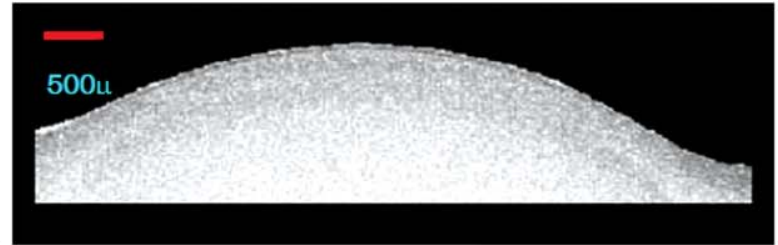

(a)

(d)
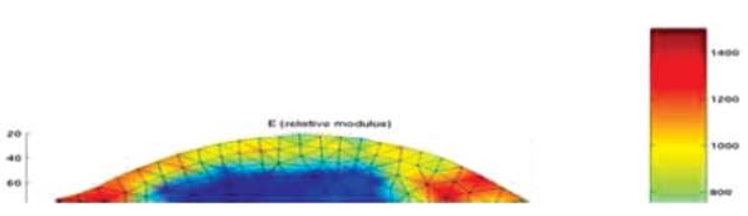

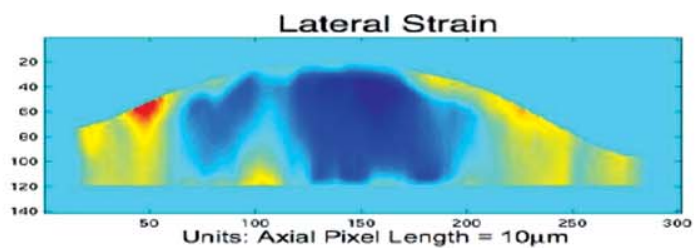

(b)

(c)
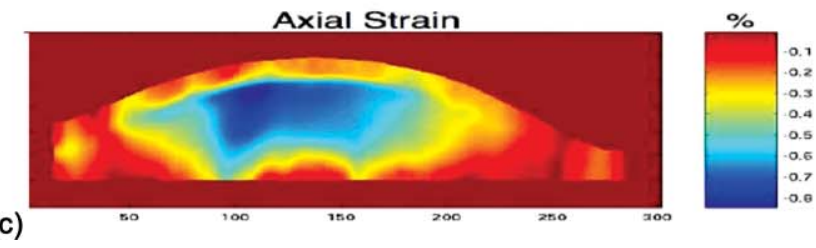

Fig. 4 (a) OCT image of porcine aorta temporally and spatially blurred with a Gaussian filter, (b) lateral strain while stretched laterally, (c) axial strain at the same state, and (d) relative modulus image (Ref. 73). Reproduced with permission from IEEE.

maximum. Radial strain can then be computed from the displacement via the following equation:

$$
\begin{aligned}
\varepsilon_{\mathrm{r}}(r, \theta) & =\frac{d}{d r} \Delta(r, \theta) \Rightarrow \varepsilon_{\mathrm{r}}, i j \\
& =\frac{1}{r_{\text {step }}}\left[\Delta\left(r_{i}, \theta_{j}\right)-\Delta\left(r_{i+j}, \theta_{j}\right)\right],
\end{aligned}
$$

where $r_{\text {step }}$ is the radial distance in pixels between the centers of subsequent windows, $\Delta$ is the displacement. The performance of the ALE algorithm applied to a simulated displacement/strain field is shown in Fig. 5. The OCT image of a vessel phantom used for simulation is shown in Fig. 5(f). The displacement field as estimated by ALE is shown in Fig. 5(a), which can be compared to the simulated input, displayed in Fig. 5(d). Strain field calculated based on ALE is shown in Fig. 5(b), which can be compared to the simulated strain field in Fig. 5(e). The peak correlation value is shown in Fig. 5(c). Comparing the pairs [Figs. 5(a), and 5(d) to 5(b), and 5(e)], one can see that the displacement and strain field are well reconstructed using the ALE algorithm.

The OCTALE method is less sensitive to motion artifacts and nonuniform catheter rotation than the traditional crosscorrelation-based method, but it is difficult to apply in practice. The applied localized force to the vascular wall at a rate of exactly half the OCT line-scan frequency must be applied, and the frequency of the applied pressure oscillation must be locked to the instrument's line rate, which brings many requirements for the load and control systems design. However, ALE expands the potential of OCT as a powerful tool for intracoronary vulnerable plaque detection.

There are still challenges faced in deployment of OCE for in vivo catheter-based imaging. The cross-correlation between frames is not suitable for cardiovascular applications because the displacement and strain patterns induced by a change in fluid pressure in a tubular geometry differ from those resulting from a mechanical force applied in a planar geometry. Additionally, when one operates an OCE probe inside a living patient, while imaging through a rotating catheter, the images
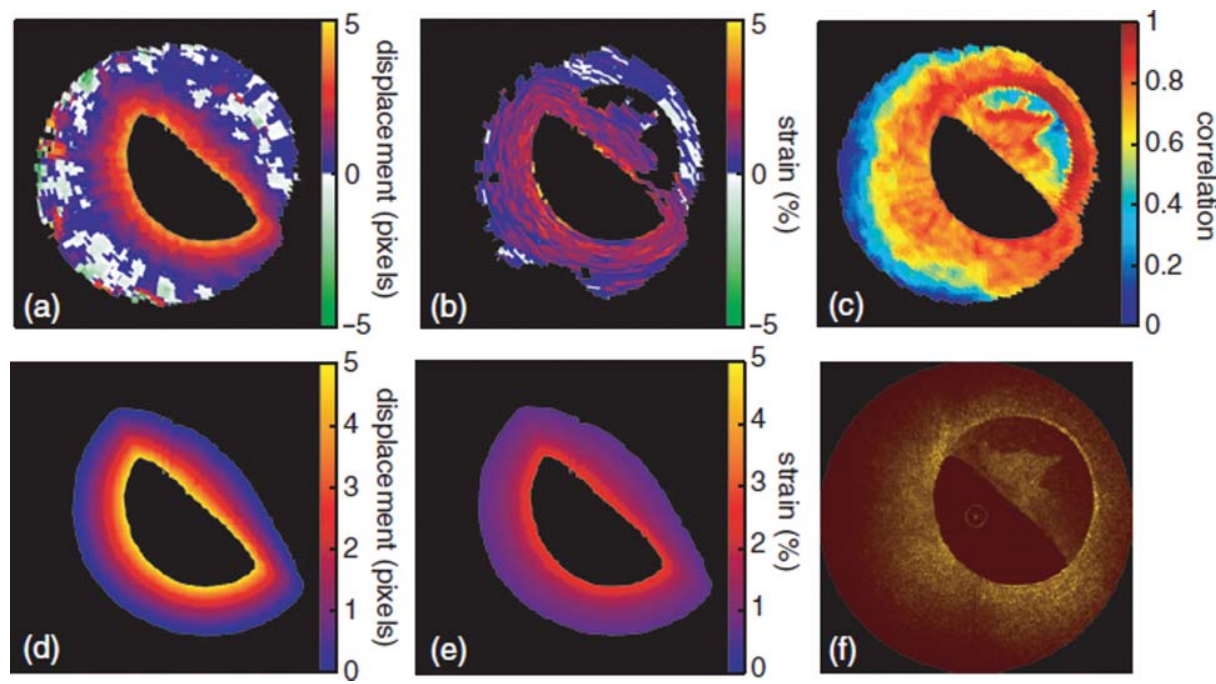

Fig. 5 (a) Displacement and (b) strain fields resulting from the ALE algorithm, and the value of (c) the correlation coefficient. The data in (a) and (b) may be compared to the simulated data $(d)$ and (e) used for input. ( $f$ ) The OCT image used for the simulation (Ref. 75). Reproduced with permission from IOP. 
and resulting stress/strain maps are more susceptible to artifacts due to motion and optical disturbances. Arteries are typically characterized by asymmetric geometries, which together with the presence of plaques with varying compositions lead to inhomogeneous stress fields in the arterial wall. Furthermore, arterial tissues exhibit a nonlinear behavior and experience high cyclic strain levels in each single cycle of a heart beat. These strain fields can vary up to $10 \%$ at the common carotid artery or carotid bifurcation. ${ }^{76,77}$ Viscoelastic models might be needed for dynamic analysis, especially for the lipid pool component of the plaques to fully characterize tissue in an effort to predict which plaques are truly vulnerable to stratify appropriate treatments or targeted cardiovascular therapies.

\section{Discussion}

The purpose of imaging elastic tissue properties is to support the detection, localization, and differential diagnosis of lesions. The quality of elastograms is determined in terms of resolution, dynamic range, and signal-to-noise ratios. These attributes are largely dependant on the ability to obtain quality estimations of tissue strain. For all elastography techniques, the resolution is determined by the underlying imaging modality. OCT has superior spatial resolution $(1-10 \mu \mathrm{m}$, in tissue), when compared to other imaging modalities, to a depth of a few millimeters resulting in a high-resolution OCE technique. The ability to provide high-resolution imaging in vivo is one of the most significant advances attributable to OCT. With even higher resolution and faster data aquisition techniques on the horizon and simplification of the procedural requirements, OCT will become more accessible to a greater number of clinical centers worldwide. ${ }^{78}$

Elastograms provide additional information of stiffness of soft tissues to OCT images, which can be especially important in medicine for early diagnosis of diseases before any morphological symptoms occur. Different schemes need to be designed for different measurement purposes. For example, breast elastography typically deals with a large single nodule that is usually $<10$ times stiffer than the normal breast tissue and static compression forces are often used. ${ }^{10}$ However, arterial elastography is more challenging than breast-tissue elastography due to the smaller length scales involved, time-varying blood flow, and presence of multiple plaques with different sizes, geometries, and compositions. Modulus of lipid pools in atherosclerotic plaques can alone span four orders of magnitude. ${ }^{79}$ Moreover, a time-varying pressure is most commonly exerted on the arterial plaques. ${ }^{47}$

Currently, there are still the following hurdles to overcome before the widespread adoption of OCE for clinically relevant in vivo applications: (i) The basic assumption for correlation-based approaches is that the speckle in the OCT image produced from the coherence volume is stable, except for a lateral translation between successive B-scans. This assumption limits these techniques to detecting motions between the successive B-scans less than the spatial coherence length of the light source used. For biomedical diagnostics, there may be potential sources of error and speckle decorrelation associated with substantial speckle pattern shifts during deformation of the soft tissues. These characteristics contribute to high noise components and must be addressed prior to the development of a robust elastography technique. (ii) The application of controlled transmural pressures is required to make accurate OCE measurements in vivo.
This will require the development of new OCT probes and can potentially be achieved with an intravascular pressure balloon or calibrated saline flushes. (iii) Kinematic and mechanical models suitable for target tissues are required. The approximation of certain tissue types being elastic and incompressible is reasonable. However, diverse soft tissues do not exhibit the linear stress-strain response. Therefore, nonlinear theory of material behavior must be implemented in order to quantify soft tissues, such as atherosclerotic or severely hypertensive arteries, where the tensile properties will not necessarily be completely uniform. (iv) Well-designed and precisely controlled experimental methods are required to investigate the anisotropic behavior of biomaterials in an effort to reduce imaging artifacts. (v) Sophisticated numerical methods, including finite element method and inverse problem method to estimate material parameters, must be continually improved to provide a model that can accurately describe specific in vivo environments. (vi) The packaging of such devices for intraluminal or internal use remains to be a critical engineering problem that must be solved before multicenter clinical testing can be performed as a first step to widespread clinical adoption of OCE. It is important to note that although these technical hurdles exist, engineering solutions are possible in the near term.

A large number of studies have demonstrated the potential of using OCT to investigate and facilitate the diagnosis of disease, direct therapeutic intervention, and image in vivo arterial pathology in humans. ${ }^{56,80-82}$ Benefiting from high-speed in vivo OCT imaging, OCE system also has great potential to measure 3-D biomechanical properties of tissues and conduct in vivo studies. With cellular-level resolution and several millimeters of imaging penetration into highly scattering tissues, OCE has the unique ability to noninvasively measure in vivo tissue biomechanical properties. Possible clinical applications of OCT elastography include differentiation of hard and soft masses during tissue biopsy, imaging of arterial plaque composition, and evaluation of wound healing. OCT elastography may also aid efforts to solve fundamental problems in biomechanics, such as the role of microscopic deformations in the development of the embryo. $^{48}$

OCE may play a major role in the biomedical imaging field specifically in intravascular and atherosclerotic tissue imaging to help the characterization of tissue biomechanical properties. Mechanical properties of vascular tissue could provide diagnostic information about a range of vascular diseases, from pulmonary hypertension to coronary atherosclerosis. The in vivo intracoronary OCE would augment the high-resolution imaging capability within formation on mechanical properties of arterial walls. Monitoring of atherosclerosis is vital for determining the effectiveness of pharmaceutical treatments that try to reduce the rupture proneness of an artery (e.g., by reducing the amount of lipid or by stiffening the lipid). A large stiffness variation near the lumen is generally assumed mechanically unstable. ${ }^{83}$ Therefore, the creation of an arterial Young's modulus image through OCE might be possible to establish a diagnostic tool for assessment of plaque vulnerability.

Because of the advantage of high resolution of OCE, additional applications are expected to be explored in areas such as tissue engineering to interpret microstructural reorganization of connective tissues during cancer progression from the macroscopic deformation patterns in viscoelastic images, molecular, 
and cellular biology, ${ }^{32}$ and shear stress over smooth muscle cells in a deformable arterial wall. ${ }^{84}$ Another application may be the material property inspection of aneurysms. ${ }^{85}$ The majority of aneurysms form in the cerebral arteries and the abdominal aorta. A cerebral aneurysm, ${ }^{86}$ as a blood-filled dilation (balloonlike bulge) in the wall of the cerebral artery, can cause devastating clinical consequences. It is widely thought that aneurysms rupture when hemodynamically induced wall stress exceeds wall strength. Hence, OCE may be very helpful for biomechanical analysis of aneurysms. ${ }^{87}$ Identification of the mechanism responsible for aneurysm rupture may be used as clinical predictors of the rupture. This area of research remains to be the focus of many studies as stroke continues to be a leading cause of morbidity and mortality in humans. As the OCE technique continues to evolve, it may elucidate a better understanding of both disease and injury and in so doing contribute to the advancement of health care.

\section{References}

1. J. Ophir, S. K. Alam, B. Garra, F. Kallel, E. Konofagou, T. Krouskop, and T. Varghese, "Elastography: ultrasonic estimation and imaging of the elastic properties of tissues," Proc. Inst. Mech. Eng. 213(H), 203233 (1999).

2. J. Ophir, I. Cespedes, H. Ponnekanti, Y. Yazdi and X. Li, "Elastography - a quantitative method for imaging the elasticity of biological tissues," Ultrason. Imaging 13(2), 111-134 (1991).

3. J. Ophir, I. Cespedes, B. Garra, H. Ponnekanti, Y. Huang, and N. Maklad, "Elastography: ultrasonic imaging of tissue strain and elastic modulus in vivo," Eur. J. Ultrasound 3, 49-70 (1996).

4. T. Gambichler, G. Moussa, M. Sand, D. Sand, P. Altmeyer, and K. Hoffmann, "Applications of optical coherence tomography in dermatology," J. Dermatol. Sci. 40(2), 85-94 (2005).

5. C. L. de Korte, A. F. W. Van Der Steen, E. I. Cespedes, G. Pasterkamp, S. G. Carlier, F. Mastik, A. H. Schoneveld, P. W. Serruys, and N. Born, "Characterization of plaque components and vulnerability with intravascular ultrasound elastography," Phys. Med. Biol. 45, 14651475 (2000).

6. T. A. Krouskop, T. M. Wheeler, F. Kallel, B. S. Garra, and T. Hall, "Elastic moduli of breast and prostate tissues under compression," Ultrason. Imaging 20(4), 260-274 (1998).

7. X. Wen and S. E. Salcudean, "Power strain imaging based on vibroelastography techniques," Proc. SPIE 6513, 65130I (2007).

8. E. Turgay, S. Salcudean, and R. Rohling, "Identifying the mechanical properties of tissue by ultrasound strain imaging," Ultrasound Med. Biol. 32(2), 221-235 (2006).

9. M. Friedrich-Rust, M. F. Ong, S. Martens, C. Sarrazin, J. Bojunga, S. Zeuzem, and E. Herrmann, "Performance of transient elastography for the staging of liver fibrosis: a meta-analysis," Gastroenterology 134(4), 960-974 (2008).

10. K. M. Hiltawsky, M. Kruger, C. Starke, L. Heuser, H. Ermert, and A. Jensen, "Freehand ultrasound elastography of breast lesions: clinical results," Ultrasound Med. Biol. 27(11), 1461-1469 (2001).

11. A. Chakraborty, G. Berry, J. Bamber, and N. Dorward, "Intraoperative ultrasound elastography and registered magnetic resonance imaging of brain tumours: a feasibility study," Ultrasound 14(1), 4349 (2006).

12. A. A. Oberai, N. H. Gokhale, S. Goenezen, P. E. Barbone, T. J. Hall, A. M. Sommer, and J. Jiang, "Linear and nonlinear elasticity imaging of soft tissue in vivo: demonstration of feasibility," Phys. Med. Biol. 54, 1191-1207 (2009).

13. K. Han-Jo, T. Wei, R. A. Stack, and S. A. Boppart, "Optical coherence elastography of developing biological tissues," Proc. SPIE 5690, 187194 (2005).

14. J. M. Schmitt, "OCT elastography: imaging microscopic deformation and strain of tissue," Opt. Express 3, 199-211 (1998).

15. V. Yang, N. Munce, J. Pekar, M. Gordon, S. Lo, N. Marcon, B. Wilson, and I. Vitkin, "Novel micromachined array tip for multi- focus fiber-based optical coherence tomography," Opt. Lett. 29, 1754 1756 (2004).

16. A. Baghani, H. Eskandari, S. Salcudean, and R. Rohling, "Measurement of viscoelastic properties of tissue-mimicking material using longitudinal wave excitation," IEEE Trans. Ultrason. Ferroelect. Frequency Control 56(7), 1405-1418 (2009).

17. K. Kumar, M. E. Andrews, V. Jayashankar, A. K. Mishra, and S. Suresh, "Measurement of viscoelastic properties of polyacrylamidebased tissue-mimicking phantoms for ultrasound elastography applications," IEEE Trans. Instrum. Meas. 59, 1224-1232 (2010).

18. S. H. Cho, J. Y. Lee, J. K. Han, and B. I. Choi, "Acoustic radiation force impulse elastography for the evaluation of focal solid hepatic lesions: preliminary findings," Ultrasound Med. Biol. 36, 202-208 (2010).

19. R. Sinkus, M. Tanter, S. Catheline, J. Lorenzen, C. Kuhl, and E. Sondermann, "Imaging anisotropic and viscous properties of breast tissue by magnetic resonance-elastography," Magn. Reson. Med. $\mathbf{5 3}$, 372-387 (2005).

20. A. D. Ieva, F. Grizzi, E. Rognone, Z. T. H. Tse, T. Parittotokkaporn, F. R. y. Baena, M. Tschabitscher, C. Matula, S. Trattnig, and R. R. y. Baena, "Magnetic resonance elastography: a general overview of its current and future applications in brain imaging," Neurosurg. Rev. 33, $137-145$ (2010)

21. F. S. Foster, C. J. Pavlin, K. A. Harasiewicz, D. A. Christopher, and D. H. Turnbull, "Advances in ultrasound biomicroscopy," Ultrasound Med. Biol. 26(1), 1-27 (2000).

22. M. J. Thali, R. Dirnhofer, R. Becker, W. Oliver, and K. Potter, "Is 'virtual histology' the next step after the 'virtual autopsy'? Magnetic resonance microscopy in forensic medicine," Magn. Reson. Imaging 22, 1131-1138 (2004).

23. J. M. Schmitt, X. Bao, and S. Xiao, "Micro-elastography of tissue with OCT," Proc. SPIE 3598, 47-55 (1999).

24. S. B. Adams Jr., M. J. Roberts, N. A. Patel, S. Plummer, J. Rogowska, D. L. Stamper, J. G. Fujimoto, and M. E. Brezinski, "The use of polarization sensitive optical coherence tomography and elastography to assess connective tissue," Conf. on Lasers and Electro-Optics, CLEO '03 1-6 June (2003).

25. M. Ford, W. J. Dupps, N. Huprikar, R. Lin, and A. M. Rollins, "OCT corneal elastography by pressure-induced optical feature flow," Proc. SPIE 6138, 1-7 (2006).

26. X. Liang, A. L. Oldenburg, V. Crecea, E. J. Chaney, and S. A. Boppart, "Optical micro-scale mapping of dynamic biomechanical tissue properties," Opt. Express 16, 11052-11065 (2008).

27. R. C. Chan, A. H. Chau, W. C. Karl, S. Nadkarni, A. S. Khalil, N. Iftimia, M. Shishkov, G. J. Tearney, M. R. Kaazempur-Mofrad, and B. E. Bouma, "OCT-based arterial elastography: Robust estimation exploiting tissue biomechanics," Opt. Express 12, 4558-4572 (2004).

28. K. C. Hilty and D. H. Steinberg, "Vulnerable plaque imaging-current techniques," J. Cardiovasc. Trans. Res. 2, 9-18 (2009).

29. F. Sharif and R. T. Murphy, "Current status of vulnerable plaque detection," Cath. Cardiovasc. Interven. 75, 135-144 (2010).

30. G. Pasterkamp and E. Falk, "Atherosclerotic plaque rupture: an overview," J. Clin. Basic Cardiol. 3, 81-86 (2000).

31. E. Falk, "Why do plaques rupture," Circulation 86, 30-42 (1992).

32. A. H. Chau, R. C. Chan, M. Shishkov, B. MacNeill, N. Iftimia, G. J. Tearney, R. D. Kamm, B. E. Bouma, and M. R. Kaazempur-Mofrad, "Mechanical analysis of atherosclerotic plaques based on optical coherence tomography," Ann. Biomed. Eng. 32(11), 1494-1503 (2004).

33. J. A. Schaar, A. F. W. v. d. Steen, F. Mastik, R. A. Baldewsing, and P. W. Serruys, "Intravascular palpography for vulnerable plaque assessment," J. Am. Coll. Cardiol. 47 (8), 86-91 (2006).

34. R. A. Baldewsing, J. A. Schaar, C. L. d. Korte, F. Mastik, P. W. Serruys, and A. F. v. d. Steen, "Intravascular ultrasound elastography: a clinician's tool for assessing vulnerability and material composition of plaques," Stud. Health Technol. Inf. 113, 75-96 (2005).

35. C. Schmitt, G. Soulez, R. L. Maurice, M.-F. Giroux, and G. Cloutier, "Noninvasive vascular elastography: toward a complementary characterization tool of atherosclerosis in carotid arteries," Ultrasound Med. Biol. 33(12), 1841-1858 (2007).

36. C. Schmitt, A. H. Henni, and G. Cloutier, "Dynamic microelastography applied to the viscoelastic characterization of a mimicking artery and a porcine aorta," in Proc. of IEEE Ultrasonics Symp., pp. 643-646 (2008). 
37. G. J. Tearney, H. Yabushita, S. L. Houser, H. T. Aretz, I.-K. Jang, K. H. Schlendorf, C. R. Kauffman, M. Shishkov, E. F. Halpern, and B. E. Bouma, "Quantification of macrophage content in atherosclerotic plaques by optical coherence tomography," Circulation 107(1), 113119 (2003).

38. B. D. MacNeill, B. E. Bouma, H. Yabushita, I.-K. Jang, and G. J. Tearney, "Intravascular optical coherence tomography: cellular imaging," Adv. Nonnucl. Imaging Technol. 12(4), 460-465 (2005).

39. C. Xu, J. M. Schmitt, S. G. Carlier, and R. Virmani, "Characterization of atherosclerosis plaques by measuring both backscattering and attenuation coefficients in optical coherence tomography," J. Biomed. Opt. 13(3), 1-8 (2008).

40. A. S. Khalil, R. C. Chan, A. H. Chau, B. E. Bouma, and M. R. K. Mofrad, "Tissue elasticity estimation with optical coherence elastography: toward mechanical characterization of in vivo soft tissue," Ann. Biomed. Eng. 33, 1631-1639 (2005).

41. A. S. Khalil, B. E. Bouma, and M. R. K. Mofrad, "A combined FEM/genetic algorithm for vascular soft tissue elasticity estimation," Cardiovasc. Eng. 6(3), 93-102 (2006).

42. W. N. Sharpe, Handbook of Experimental Solid Mechanics, Springer, Boston (2008).

43. F. Kallel and M. Bertrand, "Tissue elasticity reconstruction using linear perturbation method," IEEE Trans. Med. Imaging 15, 299-313 (1996).

44. G. R. Liu and X. Han, Computational Inverse Techniques in Nondestructive Evaluation, CRC Press, Boca Raton (2003).

45. W. Khaled, H. Ermert, S. Reichlingb, and O. T. Bruhns, "The inverse problem of elasticity: a reconstruction procedure to determine the shear modulus of tissue," in Proc. of 2005 IEEE Ultrasonics Symp, 735-738 (2005).

46. R. A. Baldewsing, M. G. Danilouchkine, F. Mastik, J. A. Schaar, P. W. Serruys, and A. F. W. v. d. Steen, "An inverse method for imaging the local elasticity of atherosclerotic coronary plaques," IEEE Trans. Inf. Technol. Biomed. 12 (3), 277-289 (2008).

47. R. Karimi, T. Zhu, B. E. Bouma, and M. R. K. Mofrad, "Estimation of nonlinear mechanical properties of vascular tissues via elastography," Cardiovasc Eng. 8, 191-202 (2008).

48. J. M. Schmitt, "Optical coherence tomography (OCT): a review," IEEE J. Sel. Top. Quantum Electron. 5(4), 1205-1215 (1999).

49. D. D. Duncan and S. J. Kirkpatrick, "Processing algorithms for tracking speckle shifts in optical elastography of biological tissues," J. Biomed. Opt. 6(4), 418-426 (2001).

50. S. J. Kirkpatrick, R. K. Wang, and D. D. Duncan, "OCT-based elastography for large and small deformations," Opt. Express 14, 11585-11597 (2006).

51. H.-J. Ko, W. Tan, R. Stack, and S. A. Boppart, "Optical coherence elastography of engineered and developing tissue," Tissue Eng. 12, 6373 (2006).

52. J. Rogowska, N. Patel, S. Plummer, and M. E. Brezinski, "Optical coherence tomographic elastography technique for measuring deformation and strain of atherosclerotic tissues," Heart 90, 556-562 (2004).

53. J. S. Steckenrider and J. W. Wagner, "Computed speckle decorrelation (CSD) for the study of fatigue damage," Opt. Lasers Eng. 22 (1), 3-15 (1995).

54. R. K. Wang, M. Zhenhe, and S. J. Kirkpatrick, "Tissue Doppler optical coherence elastography for real time strain rate and strain mapping of soft tissue," Appl. Phys. Lett. 89, 1-3 (2006).

55. S. Tang, M. Gordon, V. Yang, M. Faughnan, M. Cirocco, B. Qi, E. Seng-Yue, G. Gardiner, G. Haber, G. Kandel, P. Kortan, I. Vitkin, B. Wilson and N. Marcon, "In vivo Doppler optical coherence tomography of mucocutaneous telangiectases in hereditary hemorrhagic telangiectasia," GI Endosc. 58, 591-598 (2003).

56. V. Yang, Y. Mao, B. Standish, N. Munce, S. Chiu, D. Burnes, B. Wilson, I. Vitkin, P. Himmer, and D. Dickensheet, "Doppler optical coherence tomography with a micro-electro-mechanical membrane mirror for high speed dynamic focus tracking," Opt. Lett. 31, 1262-1264 (2006).

57. R. K. Wang, S. Kirkpatrick and M. Hinds, "Phase-sensitive optical coherence elastography for mapping tissue microstrains in real time," Appl. Phys. Lett. 90, 1-3 (2007).

58. T. Hu and J. P. Desai, in Medical Simulation, Springer, Berlin (2004), vol. 3078 , pp. $28-37$.
59. M. Bonnet and A. Constantinescu, "Inverse problems in elasticity," Inverse Prob. 21, R1-R50 (2005).

60. M. Sridhar, J. Liu, and M. F. Insana, "Elasticity imaging of polymeric media," J. Biomech. Eng. 129(2), 259-272 (2007).

61. B. Banerjee, D. Roy, and R. M. Vasu, "Efficient implementations of a pseudodynamical stochastic filtering strategy for static elastography," Med. Phys. 36(8), 3470-3476 (2009).

62. S. L. Floch, J. Ohayon, P. Tracqui, G. Finet, A. M. Gharib, R. L. Maurice, G. Cloutier, and R. I. Pettigrew, "Vulnerable atherosclerotic plaque elasticity reconstruction based on a segmentation-driven optimization procedure using strain measurements: theoretical framework," IEEE Trans. Med. Imaging 28(7), 1126-1137 (2009).

63. X. Liang, A. L. Oldenburg, V. Crecea, S. Kalyanam, M. F. Insana, and S. A. Boppart, "Modeling and measurement of tissue elastic moduli using optical coherence elastography," Proc. SPIE 6858, 1-8 (2008).

64. L. Xing and S. A. Boppart, "Dynamic optical coherence elastography and applications," Proc. SPIE 7634, 1-6 (2009).

65. X. Liang, B. W. Graf, and S. A. Boppart, "Imaging engineered tissues using structural and functional optical coherence tomography," J. Biophoton. 2, 643-655 (2009).

66. M. J. Skurczynski, F. A. Duck, J. A. Shipley, J. C. Bamber, and D. Melodelima, "Evaluation of experimental methods for assessing safety for ultrasound radiation force elastography," $\mathrm{Br}$. J. Radiol. 82, 666-674 (2009).

67. X. Liang, M. Orescanin, K. S. Toohey, M. F. Insana, and S. A. Boppart, "Acoustomotive optical coherence elastography for measuring material mechanical properties," Opt. Lett. 34, 2894-2896 (2009).

68. S. G. Adie, B. F. Kennedy, J. J. Armstrong, S. A. Alexandrov, and D. D. Sampson, "Audio frequency in vivo optical coherence elastography," Phys. Med. Biol. 54, 3129-3139 (2009).

69. Y.-P. Huang, Y.-P. Zheng, S.-Z. Wang, Z.-P. Chen, Q.-H. Huang, and Y.-H. He, "An optical coherence tomography (OCT)-based air jet indentation system for measuring the mechanical properties of soft tissues," Meas. Sci. Technol. 20, 1-11 (2009).

70. B. F. Kennedy, T. R. Hillman, R. A. McLaughlin, B. C. Quirk, and D. D. Sampson, "In vivo dynamic optical coherence elastography using a ring actuator," Opt. Express 17, 21762-21772 (2009).

71. A. L. Oldenburg and S. A. Boppart, "Resonant acoustic spectroscopy of soft tissues using embedded magnetomotive nanotransducers and optical coherence tomography," Phys. Med. Biol. 55, 1189-1201 (2010).

72. A. H. C. R. C. Chan, W. C. Karl, S. Nadkarni, A. S. Khalil, N. Iftimia, M. Shiskkow, G. J. Tearney, M. R. Kaazempur-Mofrad, and B. E. Bouma, "OCT-based arterial elastography: Robust estimation exploiting tissue biomechanics," Opt. Express 12, 4558-4572 (2004).

73. R. Karimi, R. Chan, S. Houser, B. E. Bouma, and M. R. K. Mofrad, "A novel framework for elastography and modulus estimation: Integration of tissue mechanics with imaging," in Proc. of 3rd IEEE Int. Symp. on Biomedical Imaging: From Nano to Macro, pp. 602-605 (2006).

74. G. van Soest, R. R. Bouchard, F. Mastik, N. de Jong, and A. F. W. Van Der Steen, "Robust intravascular optical coherence elastography driven by acoustic radiation pressure," Proc. SPIE 6627, 1-10 (2007).

75. G. van Soest, F. Mastik, N. de Jong, and A. F. W. Van Der Steen, "Robust intravascular optical coherence elastography by line correlations," Phys. Med. Biol. 52, 2445-2458 (2007).

76. M. Kaazempur-Mofrad, H. Younis, S. Patel, A. Isasi, C. Chung, R. Chan, D. P. Hinton, R. T. Lee, and R. D. Kamm, "Cyclic strain in human carotid bifurcation and its potential correlation to atherogenesis: idealized and anatomically realistic models," J. Eng. Math. 47, 299-314 (2003).

77. A. P. Lin, E. Bennett, L. E. Wisk, M. Gharib, S. E. Fraser, and H. Wen, "Circumferential strain in the wall of the common carotid artery: comparing displacement-encoded and cine MRI in volunteers," Magn. Reson. Med. 60, 8-13 (2008).

78. P. Barlis and J. M. Schmitt, "Current and future developments in intracoronary optical coherence tomography imaging," EuroIntervention 4, 529-534 (2008).

79. S. Hu, P. Yan, K. Maslov, J.-M. Lee, and L. V. Wang, "Intravital imaging of amyloid plaques in a transgenic mouse model using opticalresolution photoacoustic microscopy," Opt. Lett. 34(24), 3899-3901 (2009). 
80. H. Yabushita, B. E. Bouma, S. L. Houser, T. Aretz, I. K. Jang, K. H. Schlendorf, C. R. Kauffman, M. Shishkov, D. H. Kang, E. F. Halpern, and G. J. Tearney, "Characterization of human atherosclerosis by optical coherence tomography," Circulation 106(13), 1640-1645 (2002).

81. B. Standish, V. Yang, N. Munce, L. WongKeeSong, N. Buttar, K. Wang, S. Bisland, A. Lin, L. Mao, I. Vitkin, N. Marcon, and B. Wilson, "Doppler optical coherence tomography monitoring of microvascular tissue response during photodynamic therapy in an animal model of Barrett's esophagus," Gastrointest. Endosc. 66, 326-333 (2007).

82. T. Kume, T. Akasaka, T. Kawamoto, N. Watanabe, E. Toyota, Y. Neishi, R. Sukmawan, Y. Sadahira, and K. Yoshida, "Assessment of coronary arterial plaque by optical coherence tomography," Am. J. Cardiol. 97(8), 1172-1175 (2006).
83. R. A. Baldewsing, C. L. d. Korte, J. A. Schaar, F. Mastik, and A. F. W. v. d. Steen, "A finite element model for performing intravascular ultra-sound elastography of human atherosclerotic coronary arteries," Ultrasound Med. Biol. 30, 803-813 (2004).

84. M. Dabagh, P. Jalali, Y. T. Konttinen, and P. Sarkomaa, "Distribution of shear stress over smooth muscle cells in deformable arterial wall," Med. Biol. Eng. Comput. 46, 649-657 (2008).

85. J. Fromageau, S. Lerouge, R. L. Maurice, G. Soulez, and G. Cloutier, "Noninvasive vascular ultrasound elastography applied to the characterization of experimental aneurysms and follow-up after endovascular repair," Phys. Med. Biol. 53, 6475-6490 (2008).

86. J. v. Gijn, R. S. Kerr, and G. J. Rinkel, "Subarachnoid haemorrhage," Lancet 369(9558), 306-318 (2007).

87. J. C. Lasheras, "The biomechanics of arterial aneurysms," Annu. Rev. Fluid Mech. 39, 293-319 (2007). 